Original Research Paper

\title{
Comparative Analysis of the Complete Chloroplast Genome of the Alloplasmic Sunflower (Helianthus L.) Lines with Various CMS Types
}

\author{
${ }^{1}$ Kirill Azarin, ${ }^{1}$ Maxim Makarenko, ${ }^{1}$ Alexander Usatov, \\ ${ }^{1}$ Vladimir Khachumov, ${ }^{1}$ Alexey Kovalevich, ${ }^{2}$ Oleg Gorbachenko and ${ }^{3}$ Vera Gavrilova \\ ${ }^{I}$ Southern Federal University, Rostov-on-Don, Russia \\ ${ }^{2} Z$ Zlhdanov Don Experiment Station, All Russia Research Institute of Oil Crops,pos. Oporny, Rostov region, Russia \\ ${ }^{3}$ N. I. Vavilov Institute of Plant Genetic Resources, Saint Petersburg, Russia
}

\author{
Article history \\ Received: 22-12-2017 \\ Revised: 22-02-2018 \\ Accepted: 1-03-2018 \\ Corresponding Author: \\ Kirill Azarin \\ Southern Federal University, \\ Rostov-on-Don, Russia \\ Email: sunlitart@yandex.ru
}

\begin{abstract}
The complete chloroplast genomes of sunflower fertile line HA89 and isonuclear CMS lines with four different cytoplasmic backgrounds (PET1, PET2, ANN2 and MAX) were sequenced. A total of 451 polymorphic sites, with including 58 SSRs, 317 SNPs and 76 microindels were revealed between the fertile and CMS cytotypes. Among the alloplasmic male-sterile lines, cpDNA of CMS-MAX had the largest number of polymorphisms. The lowest number of polymorphic sites was revealed in CMS-PET1. Like as CMS-PET1, CMS-PET2 was obtained as a result of interspecific crossing between $H$. petiolaris and cultivated sunflower $H$. annuus. Nevertheless, the number of INDELs and SNPs in CMS-PET2 chloroplast genome was more than 4-fold and 6.5-fold higher than that in cpDNA of CMS-PET1. The average frequency of SNPs and INDELs in the non-genic regions and genic regions were 0.0062 and 0.0046 , respectively. Increased mutation rates were found in the $p s b M-$ rpoB, rps16 intron, atpA-psbD, rps4-ndhJ and ndhc-atpE non-coding regions, as well as in the rpoC2, atpA, rbcL, $n d h F$ and $y c f 1$ genes. In addition to short insertions and deletions ranging from 1 to $5 \mathrm{bp}$, the relatively long INDELs (14-24 bp) unique for each CMS line were found. These insertions and deletions may be of use for PCR differentiation of the CMS lines due to differences in the amplicons length.
\end{abstract}

Keywords: Complete Chloroplast Genome, Alloplasmic Lines, CMS, Sunflower

\section{Introduction}

The success of a high productivity hybrids breeding with tolerant to a number of environmental factors largely depends on the genetic potential of the parental lines. In the overwhelming majority of the analysis of genetic diversity in plants, the main emphasis is on the combination of nuclear alleles (Pervaiz et al., 2015). At the same time, the potential of cytoplasmic variability and new cytoplasmic-nuclear combinations are practically not taken into account. The nuclear genome plays a significant role in the ontogenesis of plants, however, the effects of cytoplasmic genes has now been proven both on the expression of quantitative traits so on the adaptive potential of plants to extreme environmental factors (Mashkina et al., 2010). Indeed, plastid DNA, which account for only a few percent of the total cellular
DNA, is involved in the realization of vital plant functions such as photosynthesis (Jansen and Ruhlman, 2012). Nuclear and cytoplasmic genomic interactions are confirmed by studies of the simultaneously variability both organelle and nuclear DNA (Russell et al., 2003). Mitochondrial and chloroplast DNA has a much lower level of variability compared to the nuclear genome. Along with this, the reduction of genetic diversity in the process of domestication and further selection is demonstrated. So, based on the restriction site polymorphism in cpDNA of 34 wild and cultivated lines of sunflower, Rieseberg and Seiler (1990) showed the monomorphism in cultivated lines for cpDNA phenotypes. Also, based on polymorphism of microsatellite loci of chloroplast genome in six Helianthus species and 46 lines of cultivated sunflower, it was demonstrated the absent cpDNA polymorphisms 
within cultivated forms of the sunflower $H$. annuus (Markin et al., 2015). Currently, almost all commercial sunflower hybrids are obtained on the basis of CMS of only one type of PET1, which was discovered by P. Leclercq (1966) in the interspecific hybrid between $H$. petiolaris Nutt and $H$. annuus L. However, intensive use of only one CMS source makes cultivated hybrids extremely vulnerable to new strains of the pathogens (Levings, 1990; Liberatore et al., 2016). In connection with the decrease in cytoplasmic genetic diversity, accompanying the processes of domestication and artificial selection, it is necessary to introduce new plasmotype into cultivated plants. The study of the structural and functional organization of the organelle genomes is also relevant from the point of view of modern taxonomy and phylogenetics. Until now, only a few chloroplast markers are used in studies of plant diversity and phylogeny (Soejima and Wen, 2006; Schroeder et al., 2011). With the advent of NextGeneration Sequencing (NGS) technologies, a real opportunity to use complete chloroplast genomes for the taxonomic and evolutionary studies was appeared (McPherson et al., 2013). The complete sequences of cpDNA of sunflower HA89-alloplasmic lines with the types of cytoplasmic male sterility PET1, PET2, ANN2 and MAX will allow us to identify an additional source of genetic variation and make a significant contribution to the solution of the fundamental problems of biology, especially in the field of genetics and evolutionary genomics of plastids.

The aim of this study was to investigate the polymorphism of the complete chloroplast genomes of sunflower fertile line HA89 and isonuclear CMS lines with four different cytoplasmic backgrounds (PET1, PET2, ANN2 and MAX).

\section{Materials and Methods}

\section{Plant Materials}

The study was carried out on sunflower fertile line HA89 and isonuclear CMS lines with four different cytoplasmic backgrounds. The plant materials were obtained from the genetic collection of the N. I. Vavilov Institute of Plant Genetic Resources (Russia). CMS sources belonged to the following species of the genus Helianthus: H. petiolaris (PET1, PET2), H. annuus (ANN2) and H. Maximilliani (MAX).

\section{Mitochondrial DNA Extraction, Genome Library Construction and Sequencing}

First of all, from leaves of 14 day sunflower seedlings was extracted organelle fraction with reduced amount of nuclear DNA as has been described earlier (Makarenko et al., 2016). For each line we used the same quantity of leaf tissue from 5 plants. The DNA isolation from such fraction was performed with PhytoSorb kit
(Syntol, Russia), according to the manufacture's instruction. The NGS library preparation was made using 1 ng of DNA and Nextera XT DNA Library Prep Kit (Illumina, USA) (Head et al., 2014). All the preparation steps were done pursuant to manual. For the qualitative control of libraries Bioanalyzer 2100 (Agilent, USA) was used. The libraries quantitation was performed with Qubit fluorimeter (Invitrogen, USA) and qPCR (van Dijk et al., 2014). For sequencing libraries were diluted up to concentration of $8 \mathrm{pM}$. Libraries were sequenced on different sequencing platforms. Fertile line, PET1, MAX1 NGS libraries were sequenced with NextSeq 500 sequencer using High Output v2 kit (Illumina, USA). PET2 and ANN2 libraries were sequenced with HiSeq2000 platform using TruSeq SBS Kit v3-HS (Illumina, USA).

\section{Analysis of Sequence Data}

Quality of reads was determined by FastQC (http://www.bioinformatics.babraham.ac.uk). Trimming of adapter-derived and low quality (Q-score below 25) reads was performed with Trimmomatic software (Bolger et al., 2014). Using Bowtie2 tool v 2.3.3 (Langmead and Salzberg, 2012) sequencing reads were aligned to reference sequences of chloroplast (NC_007977.1) genomes from NCBI databank. Variant calling was made by samtools/bcftools software (Li, 2011) and manually revised using IGV tool (Thorvaldsdóttir et al., 2013).

\section{Results and Discussion}

About 13.2, 14.7, 9.3, 6.9 and 10.2 GB of raw DNA sequences isolated from the fertile line HA 89, CMSPET1, CMS-PET2, CMS-ANN2, CMS-MAX were obtained, respectively. The overall alignment rates for chloroplast genomes were $25-30 \%$ of total read number, depending on sample. The average read coverage was more than 1000 for chloroplast genomes. Based on aligning sequencing reads to reference sequences (NC_007977.1) we obtained the complete sequence of the chloroplast genome of the studied sunflower lines. The chloroplast genomes of the HA89, CMS-PET1, CMS-PET2, CMS-ANN2, CMS-MAX consisted of $151,094 \mathrm{bp}, 151,110 \mathrm{bp}, 151127,151147$ and 151,138 bp, respectively. The difference in size is due to the increased length of individual non-coding regions of the chloroplast genome. In most terrestrial plants, the chloroplast genome consists of a circular DNA molecule, which includes a Large Single Copy region (LSC) and a small Single Copy Region (SSC) separated by two copies of Inverted Repeats (IR). The content, order and organization of chloroplast genes are usually highly conserved, which makes chloroplast genomes invaluable for genetic and phylogenetic studies. Chloroplast genomes of the researched sunflower lines are constructed on the conservative type and contain an LSC, an SSC and 
a pair of IRs region. The lengths of the LSC regions ranged from 83,527 of fertile line to 83,605 bp of CMSANN2. While the lengths of the SSC varied in five cytotypes from 19,113 of CMS-MAX to 19,147 bp of fertile line. The IR regions are characterized by the smallest length change (Table 1). The content and order of the genes in the investigated cytotypes was identical to the previously sequenced sunflower cpDNA (NC_007977.1). Regions of high $\mathrm{G}+\mathrm{C}$ content are more sensitive to mutation (Smith et al., 2002). The total $\mathrm{G}+\mathrm{C}$ content had insignificant variations from 37.60 to $37.62 \%$ (Table 1), which is comparable with the chloroplast genomes of other Angiosperms (Jansen and Ruhlman, 2012).

Comparative chloroplast genome analyses of alloplasmic CMS lines of sunflower revealed 451 polymorphic sites. Among them, 58 sites were microsatellite loci, represented exclusively by mononucleotide repeats, are located in non-coding cpDNA regions (Table 2). Chloroplast microsatellites due to uniparental inheritance have been widely used in the analysis of genetic diversity, differentiation and population structure (Provan, 2000; Flannery et al., 2006). Also, 317 SNPs and 76 microindels were identified. The ratio of SSRs, SNPs and INDEL variations were $12.9,70.2$ and $16.9 \%$, respectively. SNPs are represented by six types of nucleotide substitutions: A/G (26.9\%), A/C (14.4\%), A/T (10.5\%), T/C (29.7\%), T/G (12.5\%), G/C (6.0), whereas SSRs were as follows: (A) 6-30 (46.6\%), (T) 6-31 (46.6\%), (C) 6- $11(5.1 \%)$, (G) 7-9 (1.7\%). Our results are well consistent with the data from other studies that chloroplast microsatellite loci are mainly represented by short polyT and polyA repeats, which in turn makes a significant contribution to the prevalence of AT nucleobases (62.38-62.40\%). As is typical for other flowering plants (Ni et al., 2016; Shen et al., 2017), the most number of polymorphic sites is located in large single copy region; the lowest number of polymorphisms is located in inverted repeat region of chloroplast genomes. Of the 317 SNPs detected, 120 were located in the coding regions. Moreover, 59 substitutions were nonsynonymous (Table 3). The highest number of nonsynonymous SNPs was identified in CMS-MAX (36 substitutions), whereas the lowest value of substitutions was in CMS-PET1 ( 3 substitutions). 21 and 23 nonsynonymous substitutions were identified in CMS-PET2 and CMS-ANN2 cpDNA, respectively. Chloroplast genome of the HA89 fertile line was largely similar to the reference sequence (NC_007977.1). Only 2 INDELs in coding regions and $1 \mathrm{SNP}, 4$ INDELs in intergenic regions were identified in the fertile line (Table 4). Among the alloplasmic male-sterile lines, cpDNA of CMS-MAX had the largest number of polymorphisms in comparison with the reference sequence. The lowest number of polymorphisms was revealed in CMS-PET1 (23 SNPs and 10 indel mutations throughout the genic and non-genic regions). Like as CMS-PET1, CMS-PET2 was obtained as a result of interspecific crossing between $H$. petiolaris and cultivated sunflower $H$. annuus. It is interesting to note, that the number of INDELs and SNPs in CMS-PET2 chloroplast genome were more than 4fold and 6.5-fold higher than that in cpDNA of CMSPET1. Also, the high frequency of SNPs and indel mutations was detected in the CMS-ANN2 cytotype (Table 4). Previously, analysis of restriction site polymorphisms in the chloroplast DNA of the accessions of wild and cultivated $H$. annuus, including the lines CMS 89 and HA89, demonstrated that all accessions of domesticated sunflower had an $H$. annuus cpDNA (Rieseberg and Seiler, 1990). The authors explained the absence of $H$. petiolaris cDNA in cultivated sunflower in that the original $H$. petiolaris source for CMS 89 was a hybrid or introgressive population of $H$. annuus and $H$. petiolaris. This was subsequently confirmed by the analysis of seven individuals from the source population of $H$. petiolaris, where all seven accessions were morphologically $H$. petiolaris but had the cpDNA of $H$. annuus (Rieseberg and Seiler, 1990). Also, the high frequency of SNPs and INDEL mutations was detected in the CMS-ANN2 cytotype (Table 4 ). The ANN2 cytotype was derived from a crossing between wild and cultivated sunflower H. annuus (Serieys, 1984; Skoric et al., 2012). Previously, the comparative analysis of the complete chloroplast genomes of cultivated and wild sunflower $H$. annuus revealed only 43 variant sites, including 22 SNPs and 21 polymorphic SSR loci (Makarenko et al., 2016).

The average frequency of SNPs and INDELs in the intergenic regions was 0.0062 . Herewith, a twofold increase in frequency was identified in the intergenic region between the $p s b I$ and pet $N$ genes of the CMSMAX line. In addition, the frequency was higher in such non-genic regions as the $p s b M-r p o B$, atpA-psbD, rps4$n d h J, n d h c-a t p E$ and rps16 intron (Fig. 1).

Table 1: The features of chloroplast genomes of sunflower fertile line HA89 and 4 alloplasmic CMS lines

\begin{tabular}{llllll}
\hline Type of cytoplasm & Size $(\mathrm{bp})$ & LSC $(\mathrm{bp})$ & SSC $(\mathrm{bp})$ & IR $(\mathrm{bp})$ & GC Content $(\%)$ \\
\hline Fertile line & 151,094 & 83,527 & 19,147 & $24,210 \times 2$ & 37.62 \\
CMS- PET1 & 151,110 & 83,545 & 19,145 & $24,210 \times 2$ & 37.61 \\
CMS-PET2 & 151,127 & 83,565 & 19,138 & $24,212 \times 2$ & 37.60 \\
CMS-ANN2 & 151,147 & 83,605 & 19,118 & $24,212 \times 2$ & 37.61 \\
CMS-MAX & 151,138 & 83,601 & 19,113 & $24,212 \times 2$ & 37.61 \\
\hline
\end{tabular}


Table 2: Polymorphic microsatellite loci in the chloroplast genomes of the sunflower alloplasmic CMS lines

\begin{tabular}{|c|c|c|c|c|c|c|c|c|}
\hline $\begin{array}{l}\text { Position in } \\
\text { reference genome }\end{array}$ & Repeat MotifRef & $\mathrm{RF}$ & PET1 & PET2 & ANN2 & MAX1 & Localization & Region \\
\hline 206 & (A) 11 & & & (A) 9 & (A) 9 & (A) 9 & $r p l 2-p s b A$ & LSC \\
\hline 370 & (A) 7 & & & & & (A) 9 & $r p l 2-p s b A$ & LSC \\
\hline 1667 & (A) 6 & & & (A)7 & (A) 8 & (A) 9 & psbA-matK & LSC \\
\hline 1991 & (T) 9 & & & & & (T) 13 & psbA-matK & LSC \\
\hline 2032 & (T) 12 & & & $(\mathrm{~T}) 9$ & $(\mathrm{~T}) 14$ & (T) 10 & psbA-matK & LSC \\
\hline 3953 & $(\mathrm{~T}) 7$ & & & & & $(\mathrm{~T}) 8$ & matk-rps 16 & LSC \\
\hline 4437 & (A) 7 & & & & (A) 6 & & matk-rps 16 & LSC \\
\hline 4788 & (A) 8 & & & & & (A) 9 & matk-rps 16 & LSC \\
\hline 5450 & (C) 11 & (C) 9 & (C) 8 & (C) 10 & (C) 9 & & rps16 intron & LSC \\
\hline 5692 & (T) 13 & & (T) 11 & (T) 7 & (T) 7 & $(\mathrm{~T}) 7$ & rps 16 intron & LSC \\
\hline 5718 & (C) 7 & & & & (C) 11 & (C) 8 & rps 16 intron & LSC \\
\hline 7720 & (A) 8 & & & & & (A) 9 & $r p s 16-p s b K$ & LSC \\
\hline 7944 & (T) 8 & & & $(\mathrm{~T}) 11$ & (T) 9 & & $p s b K-p s b I$ & LSC \\
\hline 8294 & (T) 8 & & & & (T) 10 & (T) 9 & $p s b K-p s b I$ & LSC \\
\hline 8552 & (A) 8 & & & (A) 7 & (A) 7 & (A) 7 & psbI-petN & LSC \\
\hline 8880 & (T) 6 & & & & & (T)7 & psbI-petN & LSC \\
\hline 9882 & (A) 8 & & (A) 9 & (A) 9 & (A) 9 & (A) 10 & psbI-petN & LSC \\
\hline 12759 & (A) 9 & & & & & (A) 10 & psbM-rpoB & LSC \\
\hline 12984 & (T) 15 & & $(\mathrm{~T}) 11$ & $(\mathrm{~T}) 9$ & (T) 10 & (T) 14 & $p s b M-r p o B$ & LSC \\
\hline 16964 & (A) 8 & & & (A) 9 & (A) 9 & & rpoCl intron & LSC \\
\hline 17424 & (G) 9 & & (G) 8 & (G)7 & (G)7 & & rpoCl intron & LSC \\
\hline 25296 & (T) 8 & & & (T) 9 & & $(\mathrm{~T}) 10$ & atpI-atpH & LSC \\
\hline 25466 & (A) 10 & & (A) 13 & (A) $21-23$ & (A)19 & (A) 20 & atpI-atpH & LSC \\
\hline 30166 & (A) 10 & & (A) 12 & & & & atpA-psbD & LSC \\
\hline 35019 & (A) 9 & & & (A) $16-17$ & (A) 16 & (A) 8 & $p s b C-p s b Z$ & LSC \\
\hline 35398 & (A) 18 & & (A) 30 & (A) 24 & (A) 30 & (A) $18-22$ & $p s b C-p s b Z$ & LSC \\
\hline 35885 & (A) 9 & & (A) 8 & (A) 7 & (A) 7 & (A) 7 & psbZ-rps 14 & LSC \\
\hline 44324 & (A) 8 & & & (A) 10 & (A) 14 & (A) 10 & $y c f 3-r p s 4$ & LSC \\
\hline 46721 & (A) 15 & & & (A) 13 & (A) 22 & (A) 11 & rps4-ndhJ & LSC \\
\hline 50049 & (T) 8 & & & & (T) 9 & & ndhc-atpE & LSC \\
\hline 50163 & (T) 10 & & $(\mathrm{~T}) 12$ & & (T) 15 & & $n d h c-a t p E$ & LSC \\
\hline 50764 & (T) 11 & & (T) 8 & $(\mathrm{~T}) 7$ & (T) 7 & $(\mathrm{~T}) 7$ & $n d h c-a t p E$ & LSC \\
\hline 51778 & (T) 10 & & (T) 9 & (T) 21 & (T) 22 & (T) 12 & $n d h c-a t p E$ & LSC \\
\hline 54313 & (A) 16 & (A) 15 & (A) 15 & (A) 17 & (A) 21 & (A) $16-17$ & $a t p B-r b c L$ & LSC \\
\hline 58257 & (A) 7 & & & & & (A) 8 & accD-psaI & LSC \\
\hline 60017 & (C) 6 & & (C) 7 & (C) 7 & & & $y c f 4-c e m A$ & LSC \\
\hline 60433 & (T) 6 & & & (T)7 & (T) 8 & $(\mathrm{~T}) 9$ & $y c f 4-c e m A$ & LSC \\
\hline 62683 & (T) 22 & & $(\mathrm{~T}) 20$ & (T) 15 & (T) 16 & & petA-psbJ & LSC \\
\hline 64145 & (A) 9 & & (A) 8 & (A) 14 & & & psbE-petL & LSC \\
\hline 64939 & (A) 23 & & & & (A) 11 & & psbE-petL & LSC \\
\hline 65714 & (T) 16 & & & & (T) 21 & $(\mathrm{~T}) 13$ & pet $G$-psaJ & LSC \\
\hline 69743 & (A) 6 & & & & & (A) 7 & clpP intron & LSC \\
\hline 69936 & (T) 10 & & & $(\mathrm{~T}) 13$ & $(\mathrm{~T}) 11$ & (T) 9 & clpP intron & LSC \\
\hline 70545 & (A) 15 & & (A) 20 & (A) 7 & (A) 7 & (A) 10 & clpP intron & LSC \\
\hline 70584 & (T) 9 & & (T) 10 & (T) 10 & & & clpP intron & LSC \\
\hline 73405 & (T) 7 & & & (T) 8 & (T) 8 & $(\mathrm{~T}) 9$ & $p s b B-p s b T$ & LSC \\
\hline 77234 & (A) 10 & & & (A) 11 & & (A) 9 & petD-rpoA & LSC \\
\hline 79932 & (T) 22 & & (T) 15 & (T) 19 & $(\mathrm{~T}) 17$ & & rps8-rpll4 & LSC \\
\hline 80456 & (T) 24 & & & & & $(\mathrm{~T}) 27$ & rpl14-rpl16 & LSC \\
\hline 82083 & (T) 8 & & & & & (T) 9 & rpl16-rps3 & LSC \\
\hline 83644 & (T) 8 & & & $(\mathrm{~T}) 9$ & $(\mathrm{~T}) 9$ & & rps19-rpl2 & IRA \\
\hline 101481 & (T) 9 & & & & & $(\mathrm{~T}) 10$ & $r r n 16-r r n 23$ & IRA \\
\hline 119344 & (T)7 & & & $(\mathrm{T}) 8$ & $(\mathrm{~T}) 8$ & & $p s a C-n d h D$ & $\mathrm{SSC}$ \\
\hline 120979 & (A) 16 & & (A) 13 & (A) 20 & (A) 19 & (A) 10 & $n d h D-\operatorname{css} A$ & $\mathrm{SSC}$ \\
\hline 122970 & (T) 13 & & (T) 12 & (T) 18 & (T) 19 & (T) 13 & ccsA-rpl32 & SSC \\
\hline 128212 & (T) 31 & & & (T) 21 & & & $n d h F-r r n 5$ & IRB \\
\hline 133146 & (A) 9 & & & & & (A) 10 & $r r n 23-r r n 16$ & IRB \\
\hline 150984 & (A) 8 & & & (A) 9 & (A) 9 & & rpl2-rps19 & IRB \\
\hline
\end{tabular}


Kirill Azarin et al. / American Journal of Biochemistry and Biotechnology 2018, 14 (1): 39.47 DOI: $10.3844 /$ ajbbsp.2018.39.47

Table 3: Nonsynonymous substitution in the chloroplast genomes of the sunflower alloplasmic CMS lines

\begin{tabular}{|c|c|c|c|c|c|c|c|}
\hline Position & Ref & PET1 & PET2 & ANN2 & MAX1 & Gene & Region \\
\hline 2984 & $\mathrm{~A}$ & & & & $\mathrm{G}$ & matk Phe195Ser & LSC \\
\hline 14496 & A & & G & & & rpoB Gln481Arg & LSC \\
\hline 17643 & A & & & & $\mathrm{C}$ & rpoC1 Met210Leu & LSC \\
\hline 17814 & $\mathrm{C}$ & & & & $\mathrm{T}$ & rpoC1 Arg267Cys & LSC \\
\hline 18307 & G & & & A & & rpoC1 Arg431Gln & LSC \\
\hline 20660 & $\mathrm{~T}$ & $\mathrm{C}$ & & & & rpoC2 Leu490Pro & LSC \\
\hline 20750 & $\mathrm{C}$ & & & $\mathrm{T}$ & & rpoC2 Thr520Ile & LSC \\
\hline 20945 & $\mathrm{~T}$ & & & $\mathrm{G}$ & & rpoC2 Leu585Arg & LSC \\
\hline 22083 & $\mathrm{C}$ & & & & A & rрoC2 Asp964Glu & LSC \\
\hline 24141 & A & $\mathrm{C}$ & & & & rps 2 Gln178His & LSC \\
\hline 28592 & A & & & & G & atpA Ile61 Val & LSC \\
\hline 41098 & G & & & & $\mathrm{T}$ & psaA Leu144Ile & LSC \\
\hline 48363 & A & & G & $\mathrm{G}$ & & ndhJ Val107Ala & LSC \\
\hline 52386 & $\mathrm{C}$ & & $\mathrm{T}$ & $\mathrm{T}$ & & atpB Gly468Ser & LSC \\
\hline 55291 & $\mathrm{~T}$ & & & & $\mathrm{~A}$ & rbcL Cys247Asn along with 55292 & LSC \\
\hline 55292 & G & & & & A & rbcL Cys247Asn along with 55291 & LSC \\
\hline 55393 & $\mathrm{~T}$ & & & & G & rbcL Ser281Ala & LSC \\
\hline 55396 & $\mathrm{C}$ & & & & A & $r b c L G \ln 282 L y s$ & LSC \\
\hline 55534 & $\mathrm{~T}$ & & & & G & rbcL Ser328Ala & LSC \\
\hline 55675 & $\mathrm{C}$ & & & & $\mathrm{A}$ & rbcL Leu375Ile along with 55677 & LSC \\
\hline 55677 & $\mathrm{G}$ & & & & A & rbcL Leu375Ile along with 55675 & LSC \\
\hline 55837 & $\mathrm{C}$ & & & & A & $r b c L$ Gln429Lys & LSC \\
\hline 55996 & G & & & & $\mathrm{T}$ & rbcL Asp482Tyr & LSC \\
\hline 57148 & A & & & & $\mathrm{C}$ & accD Glu191Ala & LSC \\
\hline 65328 & $\mathrm{C}$ & & & & $\mathrm{T}$ & petL Pro2Leu & LSC \\
\hline 78756 & $\mathrm{~T}$ & & & $\mathrm{C}$ & & rps11 Lys8Arg & LSC \\
\hline 79664 & G & & $\mathrm{T}$ & $\mathrm{T}$ & $\mathrm{T}$ & rps8 Pro72Thr & LSC \\
\hline 82899 & $\mathrm{C}$ & & & & $\mathrm{T}$ & rpl22 AlThr & LSC \\
\hline 89152 & $\mathrm{~A}$ & & & & G & ycf2 Ile1110Val & IRA \\
\hline 108400 & $\mathrm{C}$ & & G & G & & ycf1 Asn271Lys & $\mathrm{SSC}$ \\
\hline 108722 & A & & & & $\mathrm{C}$ & ycf1 Ile379Leu & $\mathrm{SSC}$ \\
\hline 109194 & $\mathrm{C}$ & & & $\mathrm{T}$ & & ycf1 Pro536Leu & $\mathrm{SSC}$ \\
\hline 109640 & $\mathrm{G}$ & & $\mathrm{A}$ & A & & ycf Asp685Asn & SSC \\
\hline 109850 & $\mathrm{C}$ & & & $\mathrm{G}$ & & ycf1 Arg755Gly & $\mathrm{SSC}$ \\
\hline 110520 & $\mathrm{C}$ & & G & $\mathrm{G}$ & & ycf1 Thr978Arg & $\mathrm{SSC}$ \\
\hline 110568 & $\mathrm{C}$ & & A & $\mathrm{A}$ & $\mathrm{A}$ & ycf1 Thr994Lys & $\mathrm{SSC}$ \\
\hline 110913 & $\mathrm{~T}$ & & & & $\mathrm{C}$ & ycf1 Val1109Ala & $\mathrm{SSC}$ \\
\hline 110918 & $\mathrm{C}$ & & A & A & A & ycf1 His1111Asn & $\mathrm{SSC}$ \\
\hline 111048 & $\mathrm{C}$ & & & & A & ycf1 Ser1154Tyr & $\mathrm{SSC}$ \\
\hline 111059 & $\mathrm{C}$ & & & G & & ycf1 Prol158Ala & SSC \\
\hline 111605 & $\mathrm{~T}$ & & G & $\mathrm{G}$ & G & ycf1 Tyr1340Asp & $\mathrm{SSC}$ \\
\hline 111659 & $\mathrm{C}$ & & $\mathrm{T}$ & $\mathrm{T}$ & $\mathrm{T}$ & ycfl Prol358Ser & $\mathrm{SSC}$ \\
\hline 111983 & $\mathrm{~A}$ & & $\mathrm{C}$ & $\mathrm{C}$ & $\mathrm{C}$ & ycf1 Ile1466Leu & $\mathrm{SSC}$ \\
\hline 114382 & $\mathrm{~T}$ & & $\mathrm{C}$ & $\mathrm{C}$ & & ndhH Val298Ala & $\mathrm{SSC}$ \\
\hline 114585 & $\mathrm{C}$ & & $\mathrm{T}$ & & & ndhH Pro366Ser & $\mathrm{SSC}$ \\
\hline 114863 & $\mathrm{C}$ & & $\mathrm{T}$ & & & ndhA Pro64Leu & $\mathrm{SSC}$ \\
\hline 120314 & $\mathrm{~T}$ & & $\mathrm{C}$ & $\mathrm{C}$ & $\mathrm{C}$ & ndhD Leu296Pro & SSC \\
\hline 121676 & A & & & & $\mathrm{C}$ & ccsA Asn 167Lys & $\mathrm{SSC}$ \\
\hline 121813 & G & & & & $\mathrm{A}$ & ccsA His122Tyr & $\mathrm{SSC}$ \\
\hline 122087 & $\mathrm{C}$ & & A & A & & ccsA Leu30Phe & $\mathrm{SSC}$ \\
\hline 125593 & $\mathrm{~T}$ & $\mathrm{C}$ & $\mathrm{C}$ & $\mathrm{C}$ & $\mathrm{C}$ & ndhF Leu477Ser & $\mathrm{SSC}$ \\
\hline 125888 & $\mathrm{~A}$ & & $\mathrm{~T}$ & & & $n d h F$ Gln573His & $\mathrm{SSC}$ \\
\hline 125944 & G & & & & A & ndhF Ser592Asn & $\mathrm{SSC}$ \\
\hline 125949 & $\mathrm{~A}$ & & $\mathrm{~T}$ & & & $n d h F$ Asn594Tyr & SSC \\
\hline 126087 & G & & $\mathrm{T}$ & $\mathrm{T}$ & $\mathrm{T}$ & ndhF Val640Phe & $\mathrm{SSC}$ \\
\hline 126194 & $\mathrm{C}$ & & & & A & ndhF Phe675Leu & $\mathrm{SSC}$ \\
\hline 145483 & $\mathrm{~T}$ & & & & $\mathrm{C}$ & ycf2 Ile1110Val & IRB \\
\hline
\end{tabular}


Table 4: Number of SNPs and INDELs identified between the complete chloroplast genome sequences of the reference sequences (NC_007977.1) and HA89-alloplasmic CMS lines

\begin{tabular}{|c|c|c|c|c|c|c|c|c|}
\hline \multirow[b]{3}{*}{ Type of cytoplasm } & \multicolumn{8}{|c|}{ Type of sequence } \\
\hline & \multicolumn{2}{|l|}{ Genic } & \multicolumn{2}{|c|}{ Intronic } & \multicolumn{2}{|c|}{ Intergenic } & \multicolumn{2}{|l|}{ Total } \\
\hline & SNP & Indel & SNP & Indel & SNP & Indel & SNP & Indel \\
\hline Fertile line & 0 & 2 & 0 & 0 & 1 & 4 & 1 & 6 \\
\hline CMS-PET1 & 9 & 2 & 4 & 0 & 10 & 8 & 23 & 10 \\
\hline CMS-PET2 & 54 & 3 & 13 & 5 & 87 & 33 & 154 & 41 \\
\hline CMS-ANN2 & 52 & 3 & 10 & 4 & 80 & 32 & 142 & 39 \\
\hline CMS-MAX & 71 & 3 & 11 & 2 & 114 & 38 & 196 & 43 \\
\hline
\end{tabular}

Table 5: The relatively long INDELs (14-24 bp) in the chloroplast genomes of the sunflower alloplasmic CMS lines

\begin{tabular}{|c|c|c|c|c|c|}
\hline Position in ref & Type & PET2 & ANN2 & MAX1 & Localization \\
\hline $23377-78$ & INS & $\begin{array}{l}\text { CTTTCTTTT } \\
\text { ATCTTTTA }\end{array}$ & & & rpoC2-rps2 \\
\hline $32066-67$ & INS & & $\begin{array}{l}\text { TACATAAAAATT } \\
\text { AGAATAGAAAG }\end{array}$ & & atpA-psbD \\
\hline 49986-50004 & DEL & & & $\begin{array}{l}\text { GTTTCCTTTT } \\
\text { GCGGGGCGT }\end{array}$ & $n d h c-a t p E$ \\
\hline $61429-30$ & INS & & & $\begin{array}{l}\text { TCCAGTAAATAA } \\
\text { CAGAATCGTGGA }\end{array}$ & cemA-petA \\
\hline 73364-65 & INS & AGGGTACCAGAGAA & & & $p s b B-p s b T$ \\
\hline $\begin{array}{l}78307-08 \\
126449-50\end{array}$ & $\begin{array}{l}\text { INS } \\
\text { INS }\end{array}$ & TTTAATTGAGTCTTTAATTCT & TCTCTTGTTTCTCTTGC & & $\begin{array}{l}\text { rpoA-rps } 11 \\
n d h F-r r n 5\end{array}$ \\
\hline
\end{tabular}

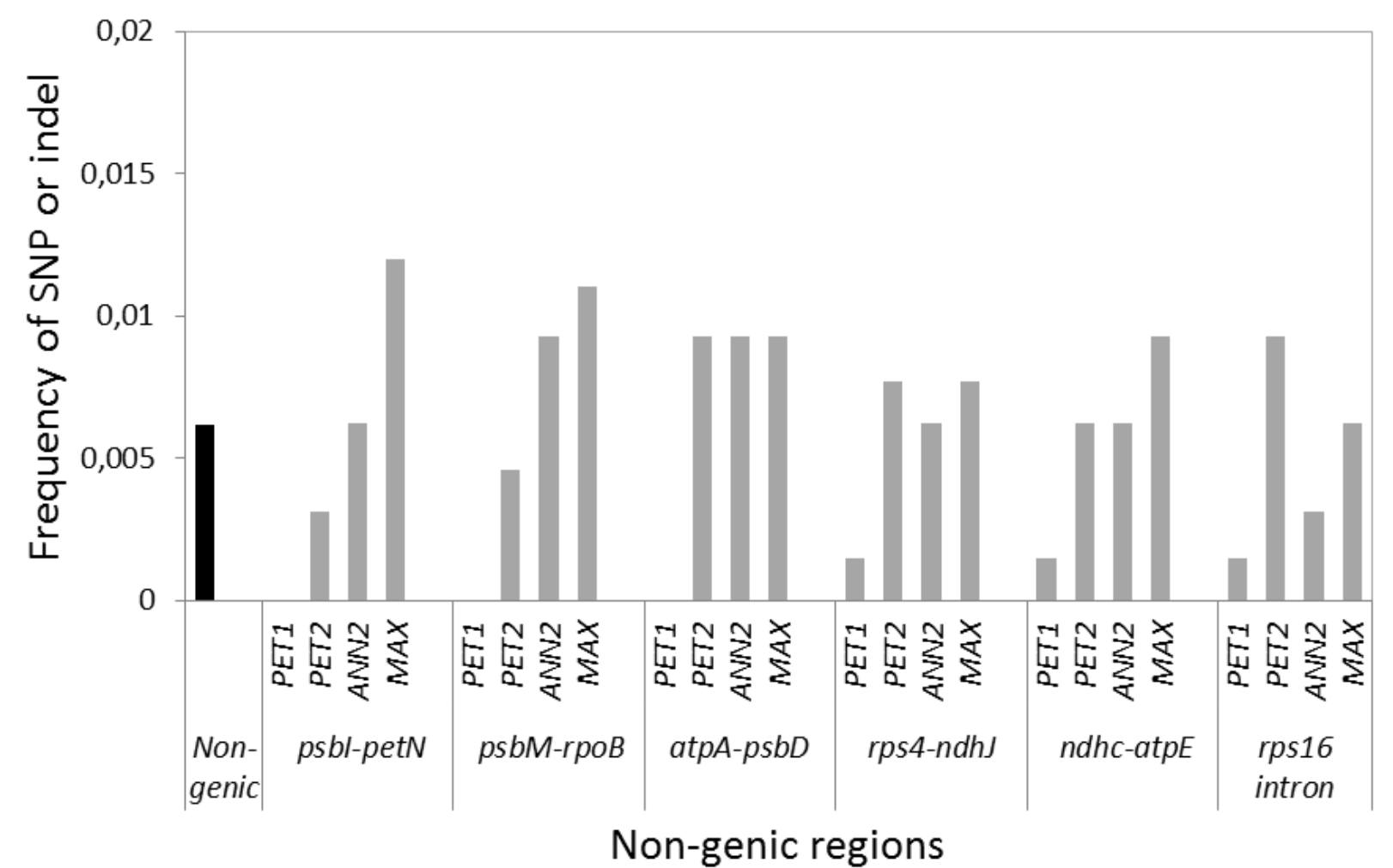

Fig. 1: Frequency of SNPs and INDELs in the highly variable non-genic regions of chloroplast genome sequences in the alloplasmic CMS lines relative to the fertile line. The grey bars indicate the frequencies of highly variable non-genic regions. The black bars indicate the average frequency of SNPs or INDELs in the non-genic regions, in total 


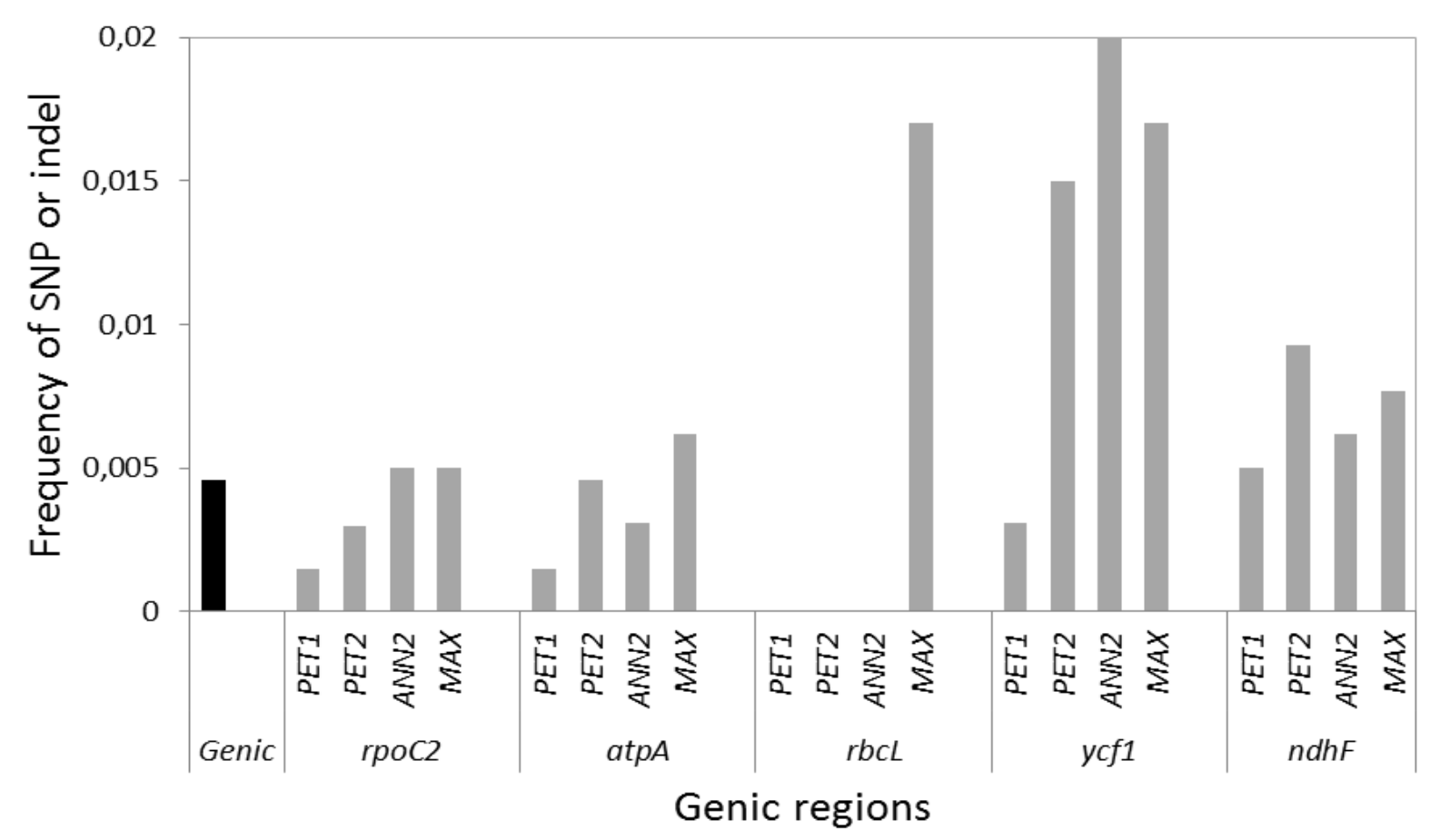

Fig. 2: Frequency of SNPs and INDELs in the highly variable genic regions of chloroplast genome sequences in the alloplasmic CMS lines relative to the fertile line. The grey bars indicate the frequencies of highly variable protein-coding genes. The black bars indicate the average frequency of SNPs or INDELs in the genic regions, in total

Meantime, the average frequency of SNPs and INDELs in the genic regions was 0.0046. Some chloroplast genes such as rpoC2, atpA, rbcL, $n d h F$ and $y c f 1$ were distinguished by a higher level of polymorphism. Interestingly, the highest increase in frequency was characterized for the $y c f l$ gene, with a 4.3-fold increases in frequency observed in the CMSANN2 line (Fig. 2). In a study devoted to a comparative analysis of the complete chloroplast genome sequences of the male-fertile line and two CMS lines of onions, it was reported that the average frequency of SNPs and INDELs in the non-genic regions was 0.0057 , while the mean frequency in the genic regions was 0.0016 (Kim et al., 2015). Among intergenic regions, the most polymorphic was the regions between the $n d h F-r p l 32$, pet $N$-psbM and trnS-trn $G$ genes. Meanwhile, as in our study, an increase in frequency of SNPs and INDELs in the genic regions was observed in the $n d h F$ (3.5-fold) and $y c f 1$ (4.2-fold times) genes (Kim et al., 2015). It was shown that the rpo genes are highly variable and reliable phylogenetic markers, effective in the reconstruction of interrelations of species belonging to the same genus (Krawczyk and Sawicki 2013). A high level of polymorphism of $n d h, r p o C 2$, rbcL and $y c f 1$ genes is also demonstrated in other studies (Wei et al., 2017; Joseph et al., 2013; Benkeblia, 2014).
In addition to short insertions and deletions (ranging from 1 to $5 \mathrm{bp}$ ) of bases in the chloroplast genome of CMS lines, the INDELs within the range of 14-24 bp in length were also found in the investigated cpDNA (Table 5). These insertions and deletions may be appropriate to use for PCR differentiation of the CMS lines due to differences in the amplicons length. Indeed, CMS-PET1 is widely used for the commercial production of F1 hybrid sunflower seeds. By now, this type of CMS has been fairly well studied. In particular, its molecular genetic bases are known and STS markers have been developed for the mitochondrial orf522 gene, which make it possible to distinguish between fertile line and sterile line which contained CMS-PET1 (Schnabel et al., 2008). The molecular basis of the CMS types like PET2, ANN2 and MAX1 is insufficiently studied, which is an obstacle to their introduction into commercial breeding. In our study, we detected INDELs specific for the chloroplast DNA of the CMS-PET2, CMS-ANN2 and CMS-MAX1 cytotypes (Table 5). The design a pair of primers for the conserved flanking regions of the INDELs will allow to development of PCR markers for identification of various types of sunflower CMS. These markers are a prerequisite for the development of highly productive heterotic sunflower hybrids on the basis of new CMS sources. 


\section{Conclusion}

The comparative analysis of complete chloroplast genomes of fertile line HA89 and 4 alloplasmic CMS lines (PET1, PET2, ANN2, MAX1) revealed a total of 451 polymorphic sites, with including 58 SSRs, 317 SNPs and 76 microindels. Chloroplast microsatellite loci are mainly represented by short polyT and polyA repeats. Of the 317 SNPs detected, 120 were located in the coding regions, 59 of these substitutions are nonsynonymous. Among the alloplasmic male-sterile lines, cpDNA of CMS-MAX was characterized by the largeast number of polymorphisms in comparison with the sequence of fertile line. The lowest number of polymorphic sites was revealed in CMS-PET1. Like as CMS-PET1, CMS-PET2 was obtained as a result of interspecific crossing between $H$. petiolaris and cultivated sunflower $H$. annuus. Nevertheless, the number of INDELs and SNPs in CMS-PET2 chloroplast genome was more than 4-fold and 6.5-fold higher than that in cpDNA of CMS-PET1. The average frequency of SNPs and INDELs in the non-genic regions and genic regions were 0.0062 and 0.0046 , respectively. Increased mutation rates were found in the $p s M M-r p o B$, rps 16 intron, atpA$p s b D$, rps4-ndhJ and $n d h c-a t p E$ non-coding regions, as well as in the $r p o C 2, a t p A, r b c L, n d h F$ and $y c f 1$ genes. In addition to short insertions and deletions ranging from 1 to $5 \mathrm{bp}$, the relatively long INDELs (14-24 bp) unique for each CMS line were found. These insertions and deletions may be of use for PCR differentiation of the CMS lines due to differences in the amplicons length.

\section{Acknowledgement}

This research was supported by a project of the Ministry Education and Science of Russia, no. 6.929.2017/4.6.

\section{Funding Information}

The funders of this manuscript were the Ministry Education and Science of Russia, no. 6.929.2017/4.6.

\section{Author's Contributions}

Kirill Azarin: Wrote the first draft of the manuscript, participated in the data analysis. Author read and approved the final manuscript.

Maxim Makarenko: Participated in the laboratory study, data analysis. Author read and approved the final manuscript.

Alexander Usatov: Designed experiments. Author read and approved the final manuscript.

Vladimir Khachumov: Participated in the laboratory study, data analysis. Author read and approved the final manuscript.
Alexey Kovalevich: Participated in the laboratory study, data analysis. Author read and approved the final manuscript.

Oleg Gorbachenko: Designed experiments. Author read and approved the final manuscript.

Vera Gavrilova: Collected and analyzed data. Author read and approved the final manuscript.

\section{Ethics}

The authors state that this article conforms to the ethical standards specified by the American Journal of Biochemistry and Biotechnology.

\section{References}

Benkeblia, N., 2014. Omics Technologies and Crop Improvement. 1st Edn., CRC Press, Taylor and Francis Group, ISBN-10: 1466586699, pp: 392.

Bolger, A.M., M. Lohse and B. Usadel, 2014. Trimmomatic: A flexible trimmer for illumina sequence data. Bioinformatics, 30: 2114-20. DOI: $10.1093 /$ bioinformatics/btu170

Flannery, M.L., F.J. Mitchell, S. Coyne, T.A. Kavanagh and J.I. Burke et al., 2006. Plastid genome characterisation in Brassica and Brassicaceae using a new set of nine SSRs. Theor. Applied Genet., 113: 1221-1231. DOI: 10.1007/s00122-006-0377-0

Head, S.R., H.K. Komori, S.A. LaMere, T. Whisenant and F. Van Nieuwerburgh et al., 2014. Library construction for next-generation sequencing: Overviews and challenges. Biotechniques, 56: 61-64. DOI: $10.2144 / 000114133$

Jansen, R.K. and T.A. Ruhlman, 2012. Plastid Genomes of Seed Plants. In: Genomics of Chloroplasts and Mitochondria, Bock, R. and V. Knoop, (Eds.), Springer Science and Business Media, Dordrecht, ISBN-10: 9400729200.

Joseph, B., J.A. Corwin, B. Li, S. Atwell and D.J. Kliebenstein, 2013. Cytoplasmic genetic variation and extensive cytonuclear interactions influence natural variation in the metabolome. Life, 2: e00776- e00776. DOI: 10.7554/eLife.00776

Kim, S., J.Y. Park and T. Yang, 2015. Comparative analysis of the complete chloroplast genome sequences of a normal male-fertile cytoplasm and two different cytoplasms conferring cytoplasmic male sterility in onion (Allium cepa L.). J Hortic Sci. Biotechnol., 90: 459-473. DOI: 10.1080/14620316.2015.11513210

Krawczyk, K. and J. Sawicki, 2013. The uneven rate of the molecular evolution of gene sequences of DNA-dependent RNA polymerase $\mathrm{i}$ of the genus Lamium L. Int. J. Mol. Sci., 14: 11376-11391. DOI: $10.3390 / \mathrm{ijms} 140611376$ 
Langmead, B. and S. Salzberg, 2012. Fast gapped-read alignment with Bowtie 2. Nat. Meth., 9: 357-359. DOI: $10.1038 /$ nmeth. 1923

Leclercq, P., 1969. Une sterilite male chez le tournesol. Ann. Amelior Plant., 19: 99-106.

Levings, C.S., 1990. The texas cytoplasm of maize: Cytoplasmic male sterility and disease susceptibility. Science, 250: 942-947. PMID: 17746917

Li, H., 2011. A statistical framework for SNP calling, mutation discovery, association mapping and population genetical parameter estimation from sequencing data. Bioinformatics, 27: 2987-2993. DOI: 10.1093/bioinformatics/btr509

Liberatore, K.L., S. Dukowic-Schulze, M.E, Miller, C. Chen and S.F. Kianian, 2016. The role of mitochondria in plant development and stress tolerance. Free Radic. Biol. Med., 100: 238-56. DOI: 10.1016/j.freeradbiomed.2016.03.033

Makarenko, M.S., A.V. Usatov, N.V. Markin, K.V. Azarin and O.F. Gorbachenko et al., 2016. Comparative genomics of domesticated and wild sunflower: Complete chloroplast and mitochondrial genomes. OnLine J. Biol. Sci., 16: 71-75. DOI: $10.3844 /$ ojbsci.2016.71.75

Markin, N.V., A.V. Usatov, M.D. Logacheva, K.V. Azarin and O.F. Gorbachenko et al., 2015. Study of chloroplast DNA polymorphism in the sunflower (Helianthus L.). Russian J. Genet., 51: 745-751. DOI: $10.1134 / \mathrm{S} 1022795415060101$

Mashkina, E.V., A.V. Usatov and M.V. Skorina, 2010. Comparative analysis of thermotolerance of sunflower chlorophyll mutants. Russ. J. Genet., 46: 178-184. DOI: 10.1134/S1022795410020079

McPherson, H., M. van der Merwe, S.K. Delaney, M.A. Edwards and R.J. Henry et al., 2013. Capturing chloroplast variation for molecular ecology studies: A simple next generation sequencing approach applied to a rainforest tree. BMC Ecol. DOI: $10.1186 / 1472-6785-13-8$

Ni, L.H., Z.L. Zhao, H.X. Xu, S.L. Chen and G. Dorje, 2016. The complete chloroplast genome of Gentiana straminea (Gentianaceae), an endemic species to the Sino-Himalayan subregion. Gene, 577: 281-288. DOI: $10.1016 /$ j.gene.2015.12.005

Pervaiz, T., X. Sun, Y. Zhang, R. Tao and J. Zhang et al., 2015. Association between chloroplast and mitochondrial DNA sequences in Chinese Prunus genotypes (Prunus persica, Prunus domestica and Prunus avium). BMC Plant Biol., 15: 4-4.

DOI: $10.1186 / \mathrm{s} 12870-014-0402-4$

Provan, J., 2000. Novel chloroplast microsatellites reveal cytoplasmic variation in Arabidopsis thaliana. Mol. Ecol., 9: 2183-2185.

DOI: $10.1046 / j .1365-294 X .2000 .105316 . x$
Rieseberg, L.H. and G.J. Seiler, 1990. Molecular evidence and the origin and development of the domesticated sunflower (Helianthus annus, Asteraceae). Econ. Bot., 44: 79-91. DOI: 10.1007/BF02860477

Russell, J.R., A. Booth, J.D. Fuller, M. Baum and S. Ceccarelli et al., 2003. Patterns of polymorphism detected in the chloroplast and nuclear genomes of barley landraces sampled from Syria and Jordan. Theor Applied Genet., 107: 413-21. DOI: $10.1007 / \mathrm{s} 00122-003-1261-9$

Schroeder, H., A. Höltken and M. Fladung, 2011. Chloroplast SNP-marker as powerful tool for differentiation of Populus species in reliable poplar breeding and barcoding approaches. BMC Proc., 5: P56- P56. DOI: 10.1186/1753-6561-5-S7-P56

Serieys, H., 1984. Wild Helianthus Species, a Potential Source of Androsterilities. 2nd Edn., Eucarpia Meeting on the Sunflower, Leningrad.

Shen, X., M. Wu, B. Liao, Z. Liu and R. Bai et al., 2017. Complete chloroplast genome sequence and phylogenetic analysis of the medicinal plant artemisia annua. Molecules. DOI: 10.3390/molecules22081330

Schnabel, U., U. Engelmann and R. Horn, 2008. Development of markers for the use of the PEF1 cytoplasm in sunflower hybrid breeding. Plant Breed, 127: $587-591$. DOI: 10.1111/j.1439-0523.2008.01516.x

Skoric, D., G. Seiler, Z. Liu, C.C. Jan and J. Miller et al., 2012. Sunflower genetics and breeding. Int. monografy. Novi Sad.

Smith, N. G. C. , M. T. Webster, and H. Ellegren, 2002. Deterministic mutation rate variation in the human genome. Genome Research, 12: 1350 - 1356. PMCID: PMC186654

Soejima, A. and J. Wen, 2006. Phylogenetic analysis of the grape family (Vitaceae) based on three chloroplast markers. Am. J. Bot., 93: 278-87. DOI: 10.3732/ajb.93.2.278

Thorvaldsdóttir, H., J.T. Robinson and J.P. Mesirov, 2013. Integrative Genomics Viewer (IGV): Highperformance genomics data visualization and exploration. Briefings Bioinform., 14: 178-192. DOI: $10.1093 / \mathrm{bib} / \mathrm{bbs} 017$

van Dijk, E.L., Y. Jaszczyszyn and C. Thermes, 2014. Library preparation methods for next-generation sequencing: Tone down the bias. Exp. Cell Res., 322: 12-20. DOI: 10.1016/j.yexcr.2014.01.008

Wei, R., Y.H. Yan, A. Harris, J.S. Kang and H. Shen et al., 2017. Plastid phylogenomics resolve deep relationships among eupolypod II ferns with rapid radiation and rate heterogeneity. Genome Biolo. Evolut., 9: 1646-1657. DOI: 10.1093/gbe/evx107 